\title{
Application of Fuzzy Linear Programming Model in Agricultural Economic Management
}

\author{
Yuping Wang \\ School of Business Administration, Xinyang Agriculture and Forestry University, Xinyang 464000, China \\ Correspondence should be addressed to Yuping Wang; 2013220002@xyafu.edu.cn
}

Received 25 November 2021; Accepted 27 December 2021; Published 24 January 2022

Academic Editor: Miaochao Chen

Copyright (C) 2022 Yuping Wang. This is an open access article distributed under the Creative Commons Attribution License, which permits unrestricted use, distribution, and reproduction in any medium, provided the original work is properly cited.

Fuzzy linear programming has a wide range of applications in agricultural economic management. In this paper, the mathematical model of fuzzy linear programming was studied in detail, and then under the restriction of elastic constraints, the $f\left(t_{i}\right)$ and $g\left(t_{0}\right)$ function expressions and their optimal solutions were obtained. Subsequently, the fuzzy linear programming was applied to the agricultural economic management of the five urban areas of Zhengzhou, and its applicability was studied. The results showed that the $f\left(t_{i}\right)$ and $g\left(t_{0}\right)$ function expressions obtained by different stretch degrees were not the same, and both had a unique optimal solution. In addition, the mathematical model based on fuzzy linear programming could well predict the pull force in agricultural economic management, so as to provide theoretical support for the application of fuzzy linear programming.

\section{Introduction}

Agriculture is not only the basis for human survival but also an important driving force for social and economic development [1]. With the ever-increasing population, food shortage has become an increasingly serious problem, which is greatly restricting social and economic development $[2,3]$. In the process of agricultural production, attention should be paid to the protection of water and soil resources. The use of water and soil resources should minimize the damage to them and maintain a constant or increased reserve of natural resources [4]. That is, while pursuing the maximization of economic benefits, we should maintain and improve the production conditions and environmental basis of natural resources. Therefore, rational development and utilization of water and land resources and maintaining agricultural economic development have become one of the keys to regional sustainable development.

Agricultural economic management requires corresponding systems and mechanisms $[5,6]$. However, from the perspective of industrial development, agricultural development tends to be consistent, and the poor utilization of resources restricts the development of agricultural economic management. In recent years, regional agriculture has tended to be homogenized, and there is generally no situation in which measures are adopted according to local conditions, which has led to imitating the models of other regions to develop their own agriculture [7]. Therefore, the efficiency of agricultural economic management is low, and traditional agriculture is widespread in many areas.

The responsibility of agricultural economic managers is to guide and encourage farmers to do the right things at the right time. Agricultural economic managers must first analyze the entire task, then assign each part of the task to farmers, organize and coordinate all farmers to participate in production, and supervise and manage farmers [8]. This requires agricultural economic managers to have the corresponding professional skills and basic knowledge. However, at this stage, China's personnel engaged in agricultural economic management in my country generally suffer from a lack of professional skills and basic knowledge. Therefore, some new technologies cannot be applied to agricultural production in time, and the speed of agricultural economic development is also restricted [9]. As a result, agricultural 
economic managers cannot innovate agricultural economic development according to the current status of local agricultural development. This situation will inevitably have a greater impact on the development of China's agricultural economy.

China's modernization started later than Europe and the United States and other developed countries. Due to the gap in productivity level, there is still a large gap between China's agricultural development level and the world advanced level, and the mechanization level is relatively low. Especially in some underdeveloped areas, production activities mainly rely on manpower. So far, a considerable part of rural areas in China have not paid enough attention to agricultural science and technology. They believe that blindly promoting agricultural mechanization and technology will cause huge losses to rural economic management and finance, resulting in the lack of financial support for the development of agricultural science and technology, resulting in a low degree of mechanization and technology. Therefore, it is not necessary to apply for the development of the agricultural economy with advanced technology. In addition, there is still much room for improvement in the introduction of agricultural talents. In real life, the frontline work in rural areas needs technical talents who are willing to contribute to the development of the agricultural economy. They should have a solid working attitude and excellent working ability [10]. However, such talents are scarce.

With the emergence of problems in agricultural economic management, people pay more and more attention to the use of mathematics to quantitatively solve various problems in the economic and management fields [11]. Among them, the proper establishment of economic mathematical models related to these problems is the key. The establishment of the mathematical model is not only the first step to solve agricultural economic and management problems with mathematics but also runs through the whole process of solving problems. There are many kinds of economic mathematical models [12]. This article mainly analyzes the fuzzy linear programming mathematical model through the development zone to achieve the development goals and discusses the standard form of the fuzzy linear programming mathematical model and the principle of the simplex solution, so as to study and solve some specific economic problems.

There are two main kinds of problems in the study of fuzzy linear programming: one is how to make an overall arrangement after a certain task is determined and try to use the least input material resources to complete the task. Second, there are already a certain amount of human and material resources, and it is important that how to arrange and use them so as to accomplish most of the tasks. In fact, these two kinds of problems are two aspects of the same problem, which is the so-called problem of seeking the best overall index of the whole problem.

Therefore, many scholars have done a lot of research work on fuzzy programming. Rojas et al. [13] first proposed the concept of decision-making in a fuzzy environment. Alemany et al. [14] studied a class of fuzzy linear programming problems with elastic constraints. Subsequently,
Mansoori et al. [15] and Crainic et al. [16] gave their respective solutions to the fuzzy linear programming problems with elastic constraints. Wang and Peng [17] obtained the optimal solution of a class of linear programming models when the parameters were fuzzy. Hamadameen and Hassan [18] considered the linear multiobjective programming problem in which the objective coefficients were fuzzy numbers and then used the function ranking method to give a solution. Nasseri et al. [19] considered fuzzy variable linear programming and gave a solution method by using the concept of fuzzy number comparison. Jiang and Chen proposed the unified principle of stochastic programming and fuzzy programming [20] and laid the foundation for optimization theory under general uncertain environments. In addition, an intuitionistic fuzzy set [21] was applied by Taghikhani et al. on the basis of a fuzzy set. It belonged to the degree and does not belong to the degree to describe fuzzy phenomena. It more objectively expressed the human thinking methods and subjective fuzzy quantities. It is a generalization of fuzzy set theory. The intuitionistic fuzzy set theory had been applied in many fields such as medical diagnosis and pattern recognition. Singh and Yadav [22] studied the optimization problem in the intuitionistic fuzzy environment, proposed a mathematical programming model about intuitionistic fuzzy sets, and gave the solution method. Luo and Zhu [23] studied the multiattribute decision-making problem with intuitionistic fuzzy sets, established several linear programming models, and gave the solution method, but the amount of calculation is relatively large. So far, fuzzy multiobjective programming has not only made a series of progress in theory but also been applied in many fields.

In view of the agricultural management problems in Zhengzhou, generalized fuzzy linear programming (GFLP) is very efficient in dealing with the problem of allowing uncertain information to appear in the optimization process and results, but it cannot solve the problem of uncertain random variables in a non-fuzzy space, nor can it consider the economic punishment after violating the policy [24-26]. This economic punishment is the first concern of policymakers. In order to solve the randomness in agricultural management, it is very efficient to deal with the "punishment" problem caused by random events. Therefore, a decision is made before the random variable is determined. After the random event occurs in the second stage, in order to make the objective function immediately, the most profitable is to "punish" the small ones, revise them, and make the second decision [27-29]. To this end, linear constraints need to be determined first if there are no constraints; the fuzzy linear gauge will become a generalized fuzzy linear programming, which will cause the objective analysis to be unclear and confusing, and the corresponding mathematical model cannot be established, so the problem cannot be solved. So we propose a generalized fuzzy linear programming model to solve the problems in agricultural economic management, so as to solve the problem of random variables caused by uncertainty and provide suggestions for agricultural economic management in Zhengzhou to optimize its planting structure and improve, and then the 
output value of agricultural production promotes the development of local agriculture.

This paper constructs and discusses a class of multiobjective fuzzy linear programming problems with elastic constraints, that is, a class of multiobjective fuzzy linear programming problems with full fuzzy coefficients except for the elastic constraint condition coefficients. Then, a method of the optimal solution is given by using the fuzzy structural element method and applied in agricultural economic management.

\section{Mathematical Model of Fuzzy Linear Programming}

Establishing a mathematical model of linear programming is to start from actual problems, grasp the main factors, determine the decision variables, find out the constraints, and establish a fuzzy linear programming model. Although many fuzzy linear programming models for economic problems have different characteristics, they all have the following three basic characteristics [30-33]:

Firstly, every seam economy problem uses a set of unknown variables $\left(x_{1}, x_{2}, \ldots, x_{n}\right)$ to represent a certain planning plan, and a set of fixed values of this set of unknown variables represents a specific plan. Moreover, the variables in these economic problems often have nonnegative requirements.

What is more, the research and resolution of these economic problems must meet certain conditions. For the fuzzy linear programming model problem, the selected conditions, namely the constraints, can be written in the form of linear equations and linear inequalities.

Finally, there are often many different options for solving these economic problems, which means that there may be many options that meet the constraints. We ask them to choose an optimal solution from them. There is a question of measurement standards, that is, according to which quantitative standards to evaluate a plan is the best, we call this quantitative standard the objective function. The objective function is determined according to the nature and requirements of economic problems. According to different research questions, the objective function is often required to take the maximum or minimum value. The objective function and constraints of each problem are linear.

In the process of agricultural economic management, it has many advantages such as dynamic tracking, freedom from linear constraints, and easy adjustment of parameters. However, it is necessary to formulate a series of socioeconomic development strategies and apply the fuzzy linear programming model to calculate the dynamic changes of each function value under different crop growth conditions. In addition, for multiobjective variables of crops, the objective function and constraint conditions are fuzzy. That is, different membership functions are used to change within a certain range to make the target value rise and fall within a certain range.

Fuzzy linear programming is to fuzzify the constraint conditions and the objective function and establish a new linear programming problem through the membership function, and its optimal solution is called the fuzzy optimal solution of the original problem.

We should determine the constraints of the model, that is, the constraints that affect the final optimization goal. This requires full consideration of various restrictive factors that affect the realization of the ultimate optimization goal, and the main restrictive factors affecting the goal planning are expressed as a constraint relation. The model established should take into account the protection of the ecological environment, economic development, and social harmony and stability in the project area. Therefore, it is necessary to establish an objective function based on the maximum economic benefit while taking into account ecological and social benefits and, finally, establish a fuzzy linear programming model. Use a two-stage solution method to solve: first, add artificial variables to the original linear programming problem, construct a fuzzy linear programming model, and then solve it; then use the simplex method to find the optimal solution of the original objective function, which is the original problem The fuzzy optimal solution of finally arrives at the optimal structural plan. The specific flow diagram is shown in Figure 1.

Because of the uncertainty of crops in their growth and harvest, the concepts of motion and fuzziness should be introduced. Agricultural economic management should focus on the growth of crops and establish elastic constraints that change within a certain range. The constraints of linear programming are rigid and inelastic. If one constraint is not satisfied, the system will have no feasible solution. For example, if a certain constraint condition $F_{i}(x) \geq b_{i}$, according to the requirements of linear programming, the optimal solution with the lowest price can be calculated only if the constraint condition is satisfied, but it may also cause price rise or imbalance of crop development completely to meet the constraint condition $F_{i}(x) \geq b_{i}$. In this case, if the system can automatically relax the constraints, it is possible to export a balanced management method for economic development. Therefore, we use fuzzy logic methods to establish a fuzzy linear programming model for the optimization matrix of agricultural economic management:

Objective function:

$$
\operatorname{MinZ}=c_{1} x_{1}+c_{2} x_{2}+\cdots+c_{n} x_{n}
$$

Constraint condition:

$$
\begin{gathered}
a_{11} x_{1}+a_{12} x_{2}+\cdots+a_{1 n} x_{1 n} \geq \approx b_{1}\left(\text { or }=, \leq \approx b_{1}\right), \\
a_{21} x_{1}+a_{22} x_{2}+\cdots+a_{2 n} x_{1 n} \geq \approx b_{2}\left(\text { or }=, \leq \approx b_{2}\right), \\
\cdots \cdots+\ldots+\cdots \cdots+a_{m n} x_{1 n} \geq \approx b_{m}\left(\text { or }=, \leq \approx b_{m}\right), \\
a_{m 1} x_{1}+a_{m 2} x_{2}+\cdots, \\
x_{2} \geq 0, \quad j=1,2, \ldots, n,
\end{gathered}
$$

where $x_{1}, x_{2}, \ldots, x_{n}$ are the decision variables, that is, various crops in the agricultural economy, $a_{i j}(i=1,2, \ldots, m$; $j=1,2, \ldots, n)$ is the output of various crops (including the limiting coefficient of the yield of a certain crop is usually 1 ). $b_{1}, b_{2}, \ldots, b_{m}$ are the original prices of the crops. $n$ is the type of crop, and $m$ is the number of constraint equations. $c_{1}, c_{2}$, 


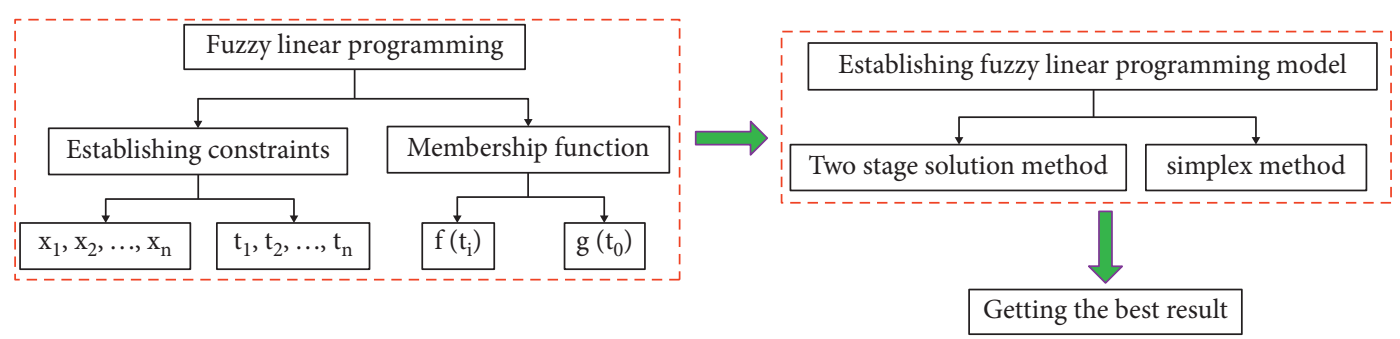

FIGURE 1: Specific flow diagram of solving fuzzy linear programming model.

$\ldots, c_{n}$ are the selling prices of each crop. $\geq \approx$ and $\leq \approx$, respectively, indicate "approximately greater than or equal" and "approximately less than or equal." Suppose $X=$ $\left\{x \mid x \in R^{n}, x \geq 0\right\}$, for each constraint $F_{i}(x) \geq \approx b_{i}$, correspondingly a fuzzy subset $D_{i}$ in $X$ corresponds to it, and its membership function is as follows:

$$
\begin{aligned}
D_{i}(x)= & f_{1}\left(\sum_{j=1}^{n} a_{\mathrm{ij}} x_{j}\right) \\
& =\left\{\begin{array}{l}
1, \quad \text { 当 } \sum_{j=1}^{n} a_{\mathrm{ij}} x_{j} \geq b_{i}, \\
1-\frac{1}{d_{i}}\left(b_{i}-\sum_{j=1}^{n} a_{\mathrm{ij}} x_{j}\right), \quad \text { 当 } b_{i}-d_{i} \leq \sum_{j=1}^{n} a_{\mathrm{ij}} x_{j}<b_{i}, \\
0, \\
\text { 当 } \sum_{j=1}^{n} a_{\mathrm{ij}} x_{j}<b_{i} .
\end{array}\right.
\end{aligned}
$$

Note that the image of $F\left(t_{i}\right)$ is shown in Figure 1, where $d_{i}$ is the appropriate degree of expansion, $d_{i} \geq 0(i=1,2, \ldots$, $m)$. Let $D=D_{1} \cap D_{2} \cap \cdots \cap D_{m} \in t(\mathrm{x})$, which is called the fuzzy constraint set of the corresponding constraint condition $A_{x} \geq \approx b(\geq 0)$. When $d_{i}=0(i=1,2, \ldots, m)$, the " $\geq$ $\approx$ " in the constraint equation degenerates to " $\geq$."

$$
\text { For the objective function }
$$

Min $Z=c_{1} x_{1}+c_{2} x_{2}+\cdots+c_{n} x_{n}$, construct the fuzzy objective set $M \in t ⿱(x)$, and its membership function is as follows:

$$
M(x)=g\left(\sum_{j=1}^{n} c_{j} x_{j}\right)=\left\{\begin{array}{l}
0, \quad \text { 当 } \sum_{j=1}^{n} c_{j} x_{j} \leq z_{0}-d_{0}, \\
\frac{1}{d_{0}}\left(z_{0}-\sum_{j=1}^{n} c_{j} x_{j}\right), \quad \text { 当 } z_{0}-d_{0}<\sum_{j=1}^{n} c_{j} x_{j} \leq z_{0}, \\
1, \quad \text { 当 } \sum_{j=1}^{n} c_{j} x_{j}>z_{0} .
\end{array}\right.
$$

$f\left(t_{i}\right)$ is mainly a multifactor function, which represents the change trend of the objective function, while $g\left(t_{0}\right)$ is the minimum value of the objective function after strictly abiding by the constraints, which reflects the lowest limit of the change of the objective function. So the image of $g\left(t_{0}\right)$ is shown in Figure 2 where $z_{0}$ is the minimum value of the objective function when the constraints are strictly observed, $d_{0}$ is the difference between the objective function value, and $Z_{0}$ after the constraints are properly scaled.

Obviously, when $D(x)=1, M(x)=0$. To make the target value smaller than $z_{0}, D(x)$ must be reduced. In order to take into account the fuzzy constraint set $D$ and the fuzzy target set $M$, the fuzzy decision $D_{F}=D \cap M$ can be used, and then the best point $x^{*}$ can be found, so that 


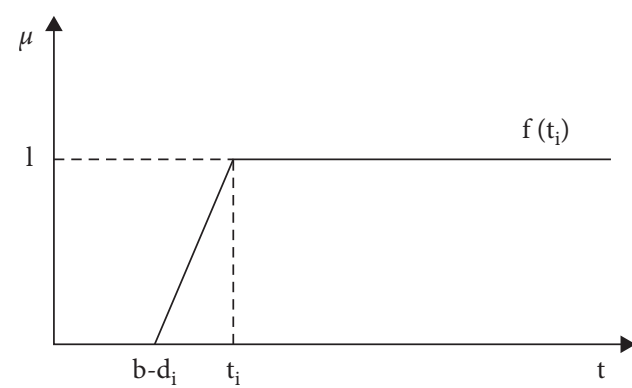

(a)

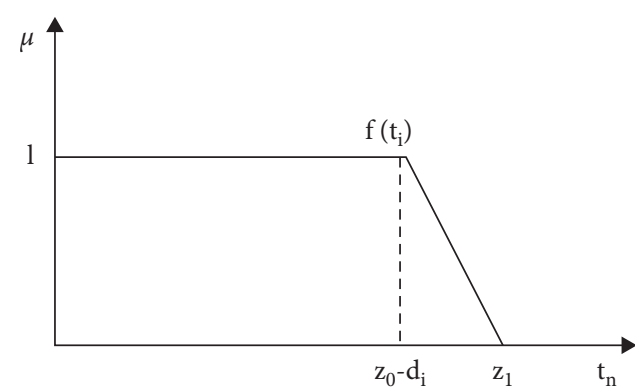

(b)

Figure 2: Function image: (a) $f\left(t_{i}\right)$ and (b) $g\left(t_{0}\right)$.

$$
D_{F}\left(x^{*}\right)=D\left(x^{*}\right) \wedge M\left(x^{*}\right)=\vee(D(x) \wedge M(x))
$$

In other words, the probability measure induced by $M$

$$
\prod(D)=\int D(x) \bullet \prod(\bullet)=D \bullet M
$$

The best point $x *$ satisfied

$$
D\left(x^{*}\right) \wedge M\left(x^{*}\right)=\prod(D)=D \bullet M
$$

Due to

$$
\begin{aligned}
D \bullet M & =\vee(D(x) \wedge M(x)) \\
& =\vee\{\lambda \mid D(x) \geq \lambda, M(x) \geq \lambda, 0 \leq \lambda \leq 1\} \\
& =\vee\left\{\lambda \mid D_{1}(x) \geq \lambda, D_{2}(x) \geq \lambda, \ldots, D_{m}(x) \geq \lambda, M(x) \geq \lambda, 0 \leq \lambda \leq 1\right\} .
\end{aligned}
$$

So the problem comes down to finding ordinary linear programming problems

$$
\begin{array}{r}
\operatorname{Min} S=\lambda, \\
1-\frac{1}{d_{i}}\left(b_{i}-\sum_{j=1}^{n} a_{\mathrm{ij}} x_{j}\right) \geq \lambda, \\
\frac{1}{d_{0}}\left(z_{0}-\sum_{j=1}^{n} c_{\mathrm{ij}} x_{j}\right) \geq \lambda, \\
0 \leq \lambda \leq 1, x_{1}, x_{2}, \ldots, x_{n} \geq 0 .
\end{array}
$$

Further transform into

$$
\begin{aligned}
\sum_{j=1}^{n} a_{\mathrm{ij}} x_{j}-d_{i} \lambda & \geq b_{i}-d_{i}, \\
\sum_{j=1}^{n} c_{j} x_{j}+d_{0} \lambda & \geq z_{0}, \\
\operatorname{Min} S & =\lambda, \\
0 & \leq \lambda \leq 1, x_{1}, x_{2}, \ldots, x_{n} \geq 0, \\
i & =1,2, \ldots, m, j=1,2, \ldots, n .
\end{aligned}
$$

Use the improved simplex method to find the optimal solution $\left(x_{1} *, x_{2} *, \ldots, x_{n}^{*}, \beth\right)$, then

$$
\prod(D)=D \bullet M=\lambda^{*}
$$

Best point formula: $x *=\left(x_{1}{ }^{*}, x_{2}{ }^{*}, \ldots, x_{n}^{*}\right)$

Minimum cost:

$$
z^{*}=\sum_{j=1}^{n} c_{j} x_{j}^{*}
$$

\section{Application of Fuzzy Linear Programming Model in Agricultural Economic Management}

Zhengzhou city is located in the southern part of the North China Plain and the lower reaches of the Yellow River. It is located in the north of central Henan Province, bordering Kaifeng in the East, Luoyang in the west, the Yellow River in the north, facing Xinxiang and Jiaozuo, and Xuchang and Pingdingshan in the south. The whole city is $135-143 \mathrm{ki}-$ lometers long from east to west and 70-78 kilometers wide from north to south, with a total area of 7446 square kilometers. In addition, Zhengzhou is located in the remaining vein of the eastern Qinling Mountains and the transition zone between the second and third geomorphic steps in China. The overall terrain is high in the southwest and low in the northeast, falling in a ladder shape. It gradually decreases from the tectonic erosion of low and medium mountains in the West and southwest to the tectonic denudation hills, 
loess hills, inclined (hillock) plains, and alluvial plains, forming a relatively complete geomorphic sequence. Meanwhile, Zhengzhou has a continental monsoon climate in the northern temperate zone, with frequent alternation of heating and cooling groups, and four distinct seasons in spring, summer, autumn, and winter. Winter is long and dry and cold; rain and snow are scarce; spring is dry and less rainy, but spring is dry, cold, warm, and more windy. What is more, summer is relatively hot, with high precipitation, and autumn has a cool climate and short time. The annual average temperature is $15.6^{\circ} \mathrm{C}$; August is the hottest, with an average monthly temperature of $25.9^{\circ} \mathrm{C}$; January is the coldest, with an average monthly temperature of $2.15^{\circ} \mathrm{C}$. The annual average rainfall is $542.15 \mathrm{~mm}$, and the frost-free period is 209 days. The annual sunshine time is about $1,869.7$ hours.

In general, Zhengzhou is an important central city, a megacity, an important national comprehensive transportation hub, a commercial and logistics center, a central city in the Central Plains urban agglomeration, and a national garden city in the central region of China. Therefore, the study of agricultural management in Zhengzhou is of great significance for the agricultural economic development of Zhengzhou, Henan Province, and even China.

Wheat, corn, and oil crops are common crops suitable for growth in Zhengzhou, and these three crops grow just in Zhengzhou all year round, which can better verify the application of fuzzy linear programming in agricultural economic management. So, in this article, the uncertainty expressed in the interval and fuzzy form in the objective function and elastic constraint conditions were used as parameters. According to the agricultural data statistics of a certain central part of Zhengzhou city, the planting goals of various crops in each district were planned, with the unit of a hectare, as shown in Table 1.

It can be seen that fuzzy linear programming could make detailed budgets for crop planting goals and achieve the set expansion according to the results of linear changes, which could reduce costs and save the land. In general, technicians would make adjustments according to specific conditions when planning and planting crops. Fuzzy linear programming provided a scientific basis for this adjustment, which was more convenient and convenient than linear programming after adjustment. And the program would also automatically take into account the balance of various crops.

Figure 3 is the function values of $f\left(t_{i}\right)$ and $g\left(t_{0}\right)$ calculated according to the fuzzy linear programming model. It can be seen that the function values of $f\left(t_{i}\right)$ and $g\left(t_{0}\right)$ obtained by fuzzy linear programming had similar changing laws and present a consistent trend. This showed that when elastic constraints were adopted, fuzzy linear programming could get the best technical parameters, which provided a certain scientific basis and data support for the application of fuzzy linear in agriculture.

In the following research, the generalized fuzzy twostage method was used to make decision support for crop allocation in the five urban areas of Zhengzhou, and the robust stepwise interactive algorithm was used to calculate the generalized fuzzy two-stage model. Based on the robust stepwise interactive algorithm, all fuzzy parameters became fuzzy intervals. Therefore, the generalized fuzzy two-stage model could be turned into an interval two-stage stochastic programming sub-model. After many cases dealing with water/air quality management and energy system management, the robust stepwise interactive algorithm handles interval two-stage stochastic programming and linear programming. The model was very efficient, so it was used to calculate the model.

The crop planting planning models of the five urban districts of Zhengzhou city under different subordination degrees were derived from a series of fuzzy interval results through the method of this paper. Therefore, the membership function of the model decision variables could be used for regression analysis based on the fuzzy interval results. Figure 3 shows the partial membership functions of fuzzy variables in different urban areas. Based on the model results, the above figure showed that the membership function of the parameters could be well adapted to linear and multiple regression. In addition, Figure 4 also provided the model target results under different membership degrees. In general, the results showed that uncertain input under different membership degrees will lead to different model system results. The upper limit of the model objective function value corresponded to favorable conditions, while the lower limit was related to the corresponding demand conditions.

In the actual problem management that the model represents, many parameters are uncertain. The crop changes in different regions in the article could be represented in the form of fuzzy sets to express the change interval of the model input under different membership degrees. At the same time, it also brings different model decision plans. Figure 5 shows the upper and lower limits of the function under different membership degrees. It could be seen that each bar represented the output of different crops in different regions under different degrees of membership. Meanwhile, the output of different crops would have different changes under different degrees of subordination. The lower limit increased with the increase of degree, but the upper limit had an opposite trend. The reason for this change was due to the ambiguity of the model input parameters. The article used an example of agricultural management in Zhengzhou to prove the practicality of the fuzzy linear programming model. We needed to plant crops in different areas to meet social and economic development. There were three crops in the study, namely, wheat, corn, and oil crops. Two different functions were obtained through the fuzzy linear programming model, and then the output of each crop was statistically analyzed to obtain the best linear fitting function. Subsequently, the upper and lower limits of the different membership degrees of crops were analyzed. Then, the optimal agricultural economic management level under the maximum profit value was obtained, which had good application value. In general, the upper and lower values represent the upper and lower limits of crops calculated by the fuzzy linear programming model, which represents the floating range of crops, which is conducive to better calculating the errors in agricultural economic management. 
TABLE 1: Crop planting objectives of each district in Zhengzhou.

\begin{tabular}{lccc}
\hline Region & & Crop planting objectives & \\
& Wheat & Corn & Oil crops \\
\hline Zhongyuan district & $(6,980.50,6,580.25)$ & $(10,158.2,14,856.5)$ & $(28,975.6,32,458.6)$ \\
Jinshui district & $(15,864.5,6,878.6)$ & $(20,156.8,25,458.6)$ & $(45,628.5,48,658.5)$ \\
Erqi district & $(10,880.5,14,680.95)$ & $(38,560.5,51,268.5)$ & $(7,485.5,6,685.4)$ \\
Gongyi city & $(20,058.5,26,585.4)$ & $(36,875.5,40,258.5)$ & $(50,258.5,54,685.5)$ \\
Xinzheng city & $(30,586.5,24,865.8)$ & $(25,687.3,30,558.5)$ & $(42,005.8,46,857.5)$ \\
\hline
\end{tabular}

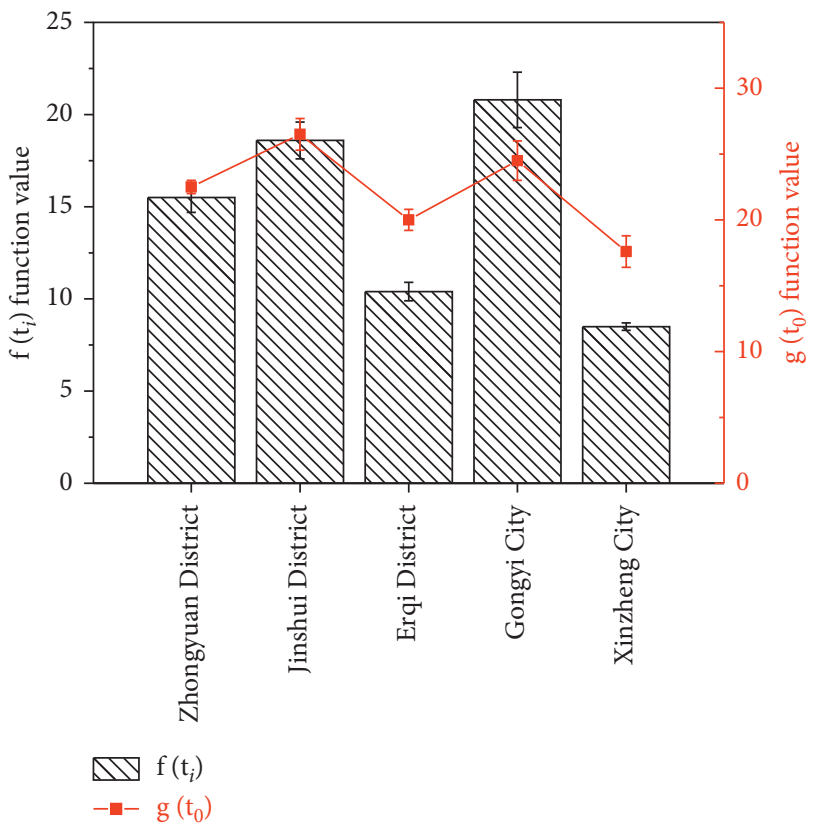

FIgURE 3: The function values of $f\left(t_{i}\right)$ and $g\left(t_{0}\right)$ calculated according to the fuzzy linear programming model.

Figure 6 is the diagram showing the relationship between the agricultural economic driving force and time between different urban areas of Zhengzhou. It can be seen that according to the agricultural economic driving force calculated by the fuzzy linear programming, as time increased, the pull force first increased, and then gradually decreased. The economic pull force was the largest at about $60 \mathrm{~d}$. In addition, the pull force between the urban areas was the same as the function value change law obtained from the fuzzy linear programming model. That is, Gongyi city had the greatest pull force, and Xinzheng city had the least pull force. The main reason should be attributed to that there were some differences in the weather environment between the two regions, and the natural weather conditions in Gongyi city were much better than those in Xinzheng city. These uncontrollable weather factors will cause the lack of driving force of the agricultural economy. At the same time, the results were also the main factors that caused the different driving forces in the urban areas. Therefore, when estimating the driving force of economic capacity, the influence of local weather conditions and other factors on the driving force should also be fully considered.

During the study period, the crops in Xinzheng city and Erqi district are very insufficient for the driving force of the agricultural economy. It shows that during the research period, Xinzheng focuses on the development of the manufacturing industry and does not pay much attention to the development of agriculture and forestry, and the ability to drive economic development is uneven. At the same time, it also explains to a certain extent that Xinzheng mainly vigorously develops the non-agricultural economy and the economic production capacity is unbalanced. The above results reveal the economic development model of Xinzheng city, and the whole economic market is dominated by manufacturing. From the result analysis, we should appropriately strengthen the management of the agricultural economy, vigorously develop the agricultural economy, and increase the driving force of the agricultural economy, so as to balance the whole industrial structure.

Figure 7 shows the relationship between the simulated function values obtained after removing the minimum and maximum driving forces and different crops. It can be seen that the yield of wheat was the lowest in the three areas, followed by corn and finally oil crops, which showed that oil crops were an important product of agricultural economic development. In my opinion, the main reason was that the yield was reduced due to the impact of the weather during the planting of wheat, and the local residents also like to 


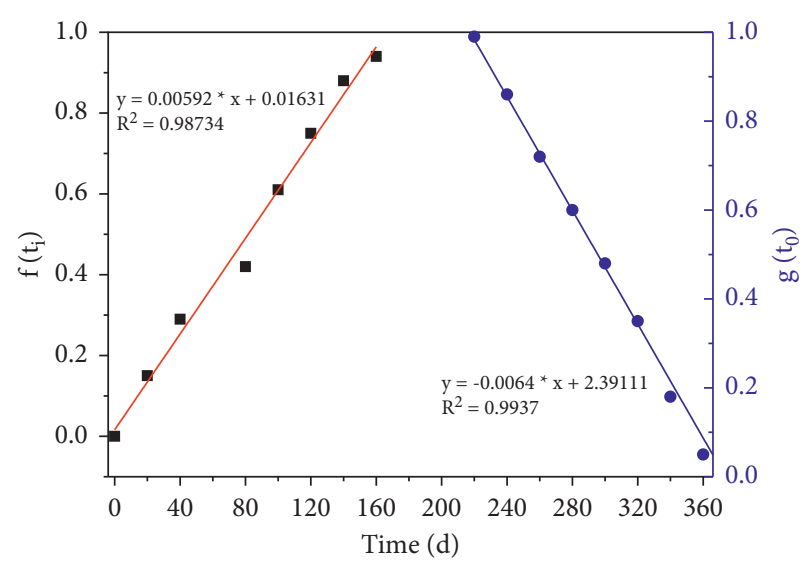

(a)

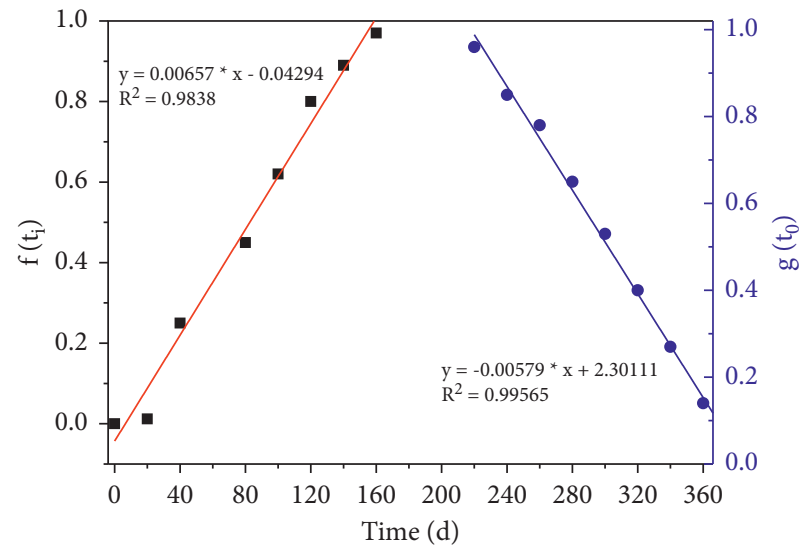

(c)

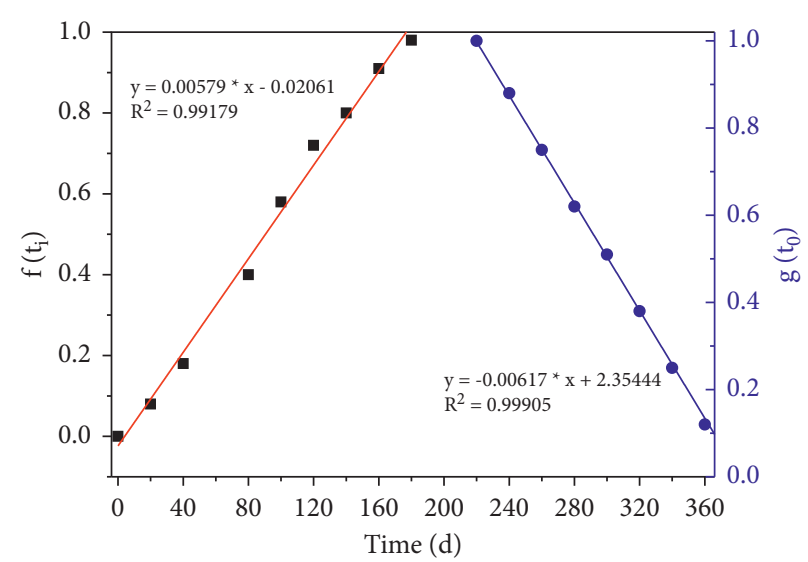

(b)

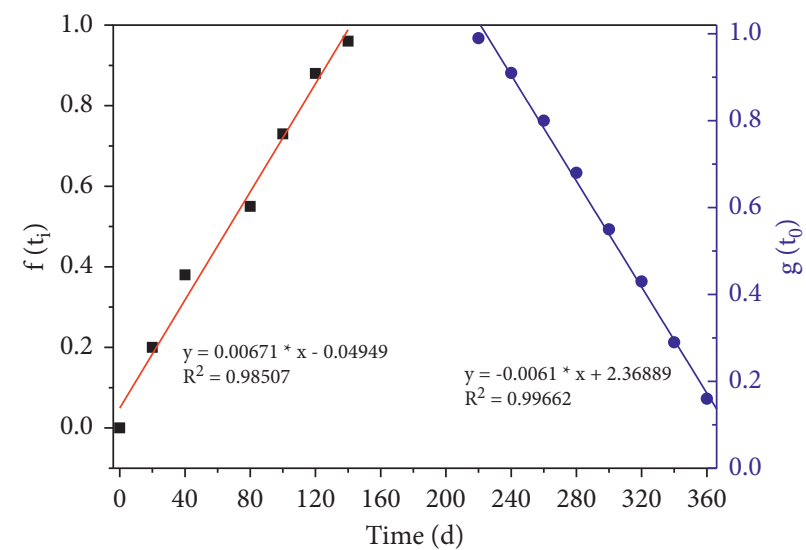

(d)

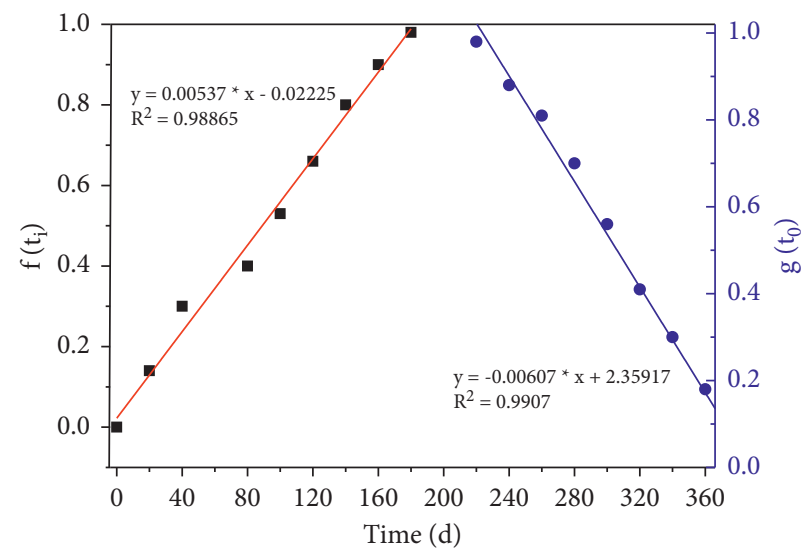

(e)

FIGURE 4: Membership functions of fuzzy variables in different urban areas: (a) Zhongyuan district, (b) Jinshui district, (c) Erqi district, (d) Gongyi city, and (e) Xinzheng city.

plant a large number of oil crops, which belonged to the special local climate. Therefore, the yield change trend of the three crops in the three areas is the same. In order to achieve the balance of agricultural economic development, the planting ratio of the three crops should be strengthened, so as to obtain the optimal crop planting proportion.

Generalized fuzzy linear programming is very efficient in solving the problem of constraint conditions with coefficients or objective functions with fuzzy intervals, but it cannot solve the problem of uncertain random variables in a non-fuzzy space, and it cannot be considered in violation of the policy. It is the first concern of policymakers for the subsequent economic punishment. Therefore, different model function values (Figure 8 ) are obtained by limiting an elastic constraint condition, and then the random variable problem in agricultural economic management is solved through the change of different calculation data. The main reason may be that the driving force of crops in Gongyi city 


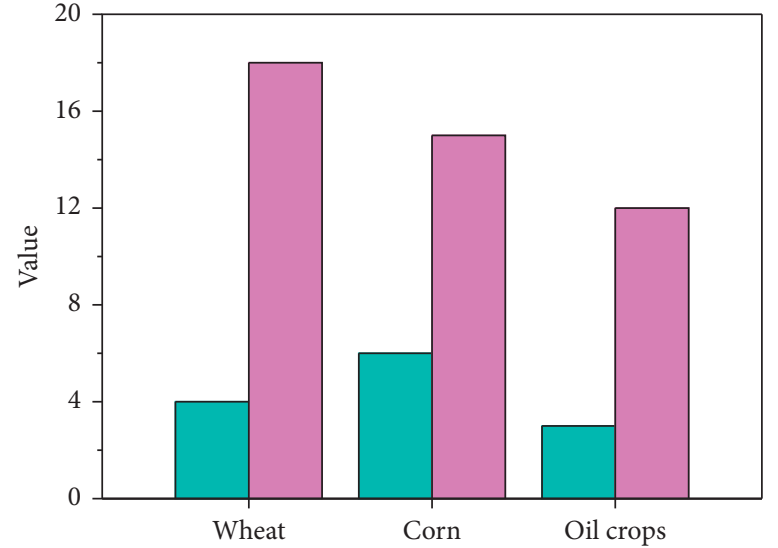

Lower limit

Upper limit

(a)

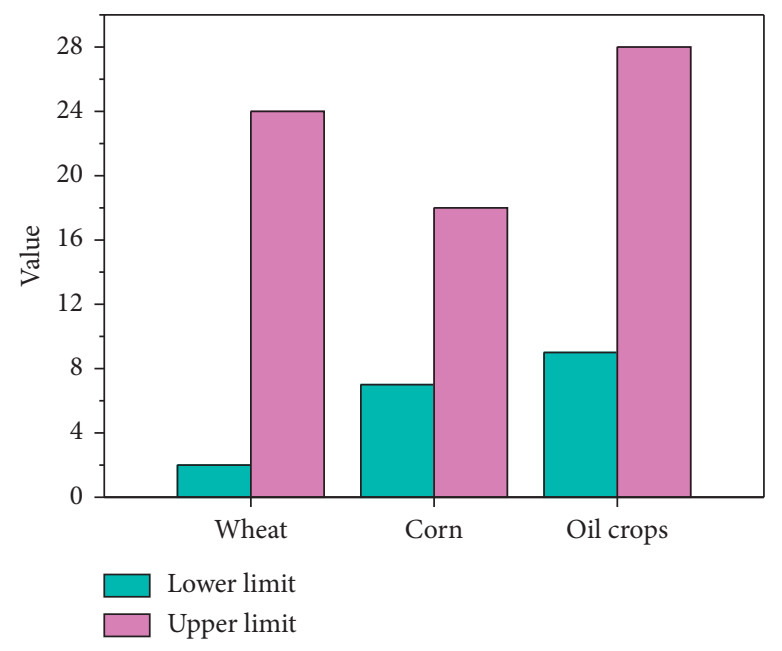

(c)

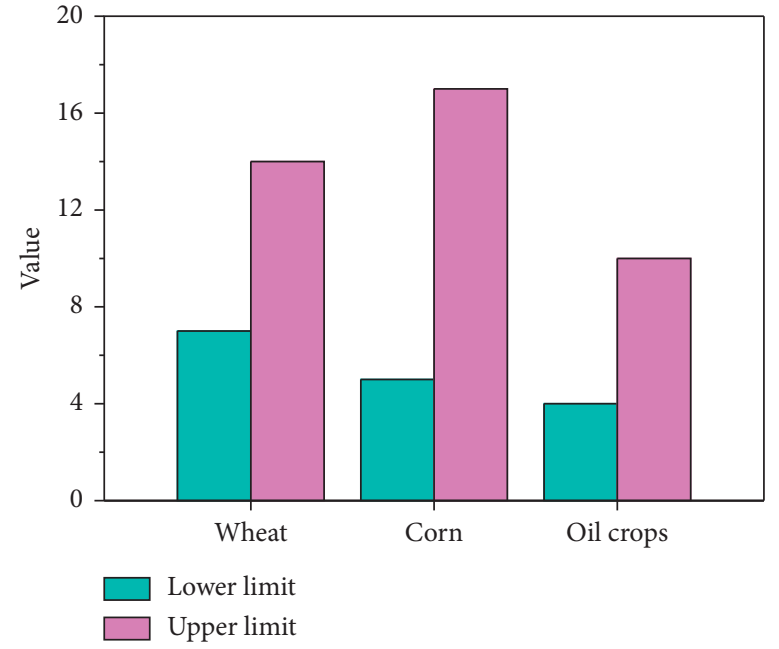

(b)

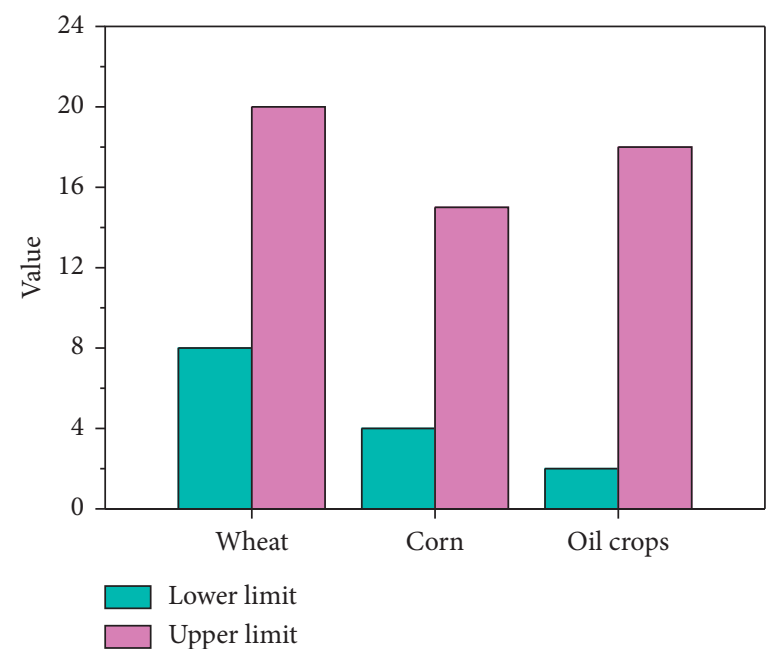

(d)

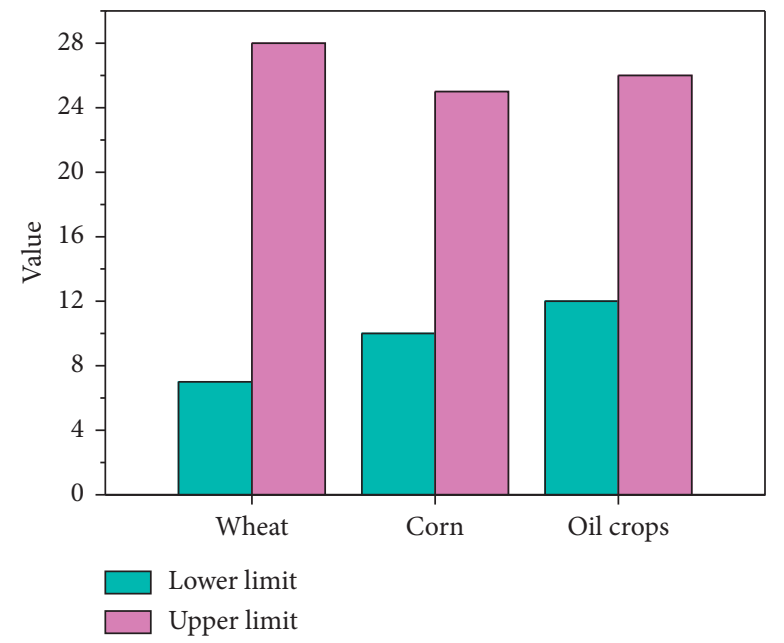

(e)

FIgURE 5: The upper and lower limits of the function under different membership degrees: (a) Zhongyuan district, (b) Jinshui district, (c) Erqi district, (d) Gongyi city, and (e) Xinzheng city. 


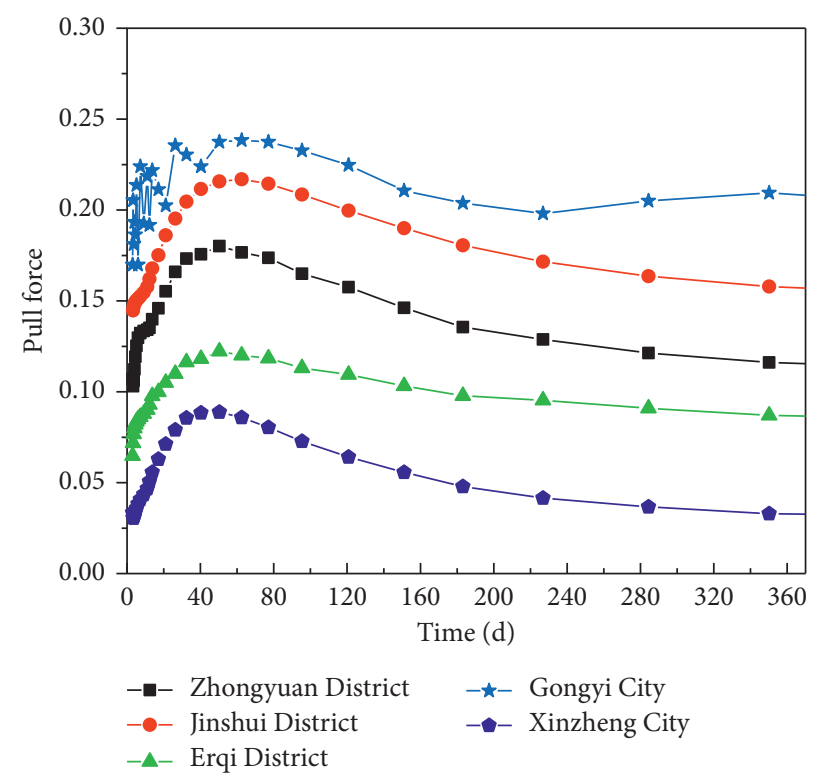

FIGURE 6: Relationship between pull force and time among urban areas.

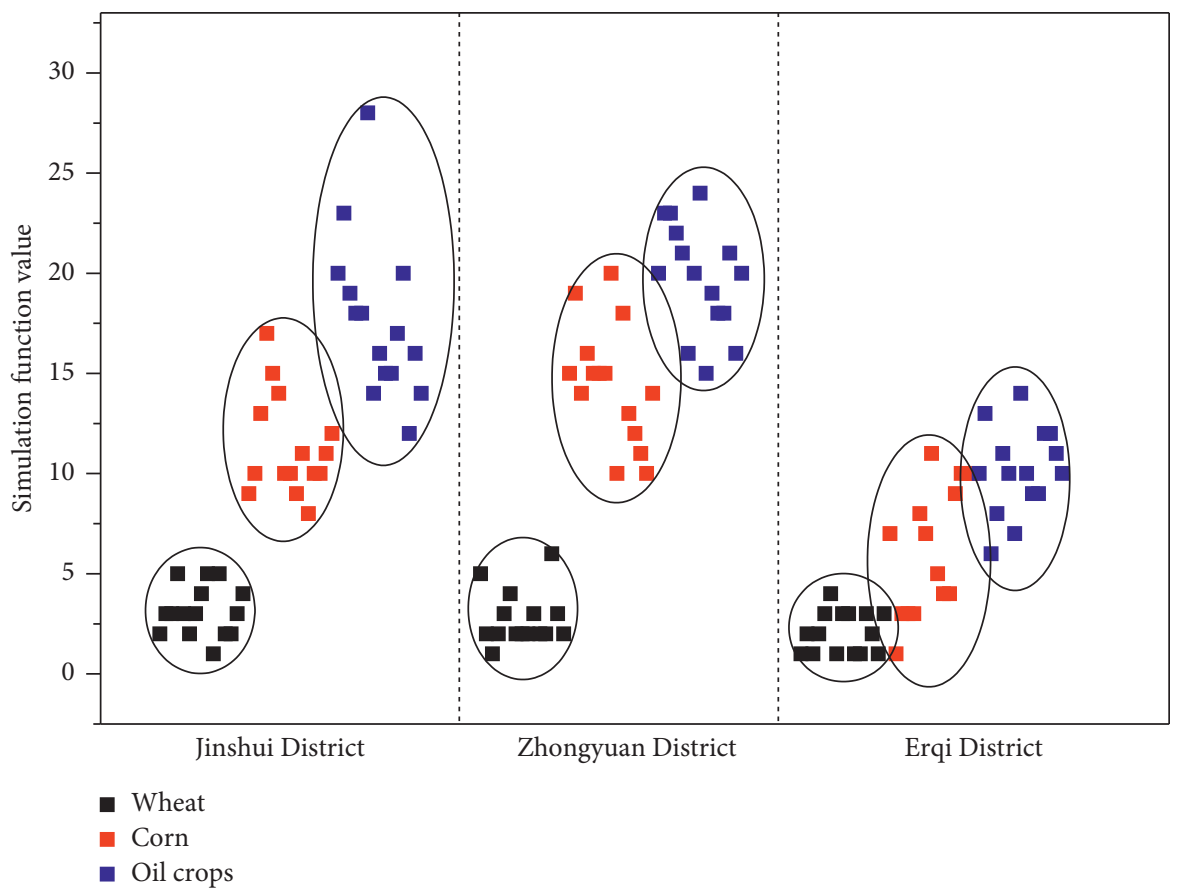

FIgURE 7: Relationship between simulation function values and different crops in three urban areas.

and Jinshui district is large, which corresponds to the results in Figure 6. In addition, as the main crop in Zhengzhou, oil crops have a large planting amount and driving force, so the function value calculated by fuzzy linear programming is also large. Aiming at the level of agricultural development between different urban areas and different crops in Zhengzhou, a generalized fuzzy linear programming problem was established to solve the allocation problem in the development of the agricultural economy, minimize the consumption of water resources and land, and ensure 

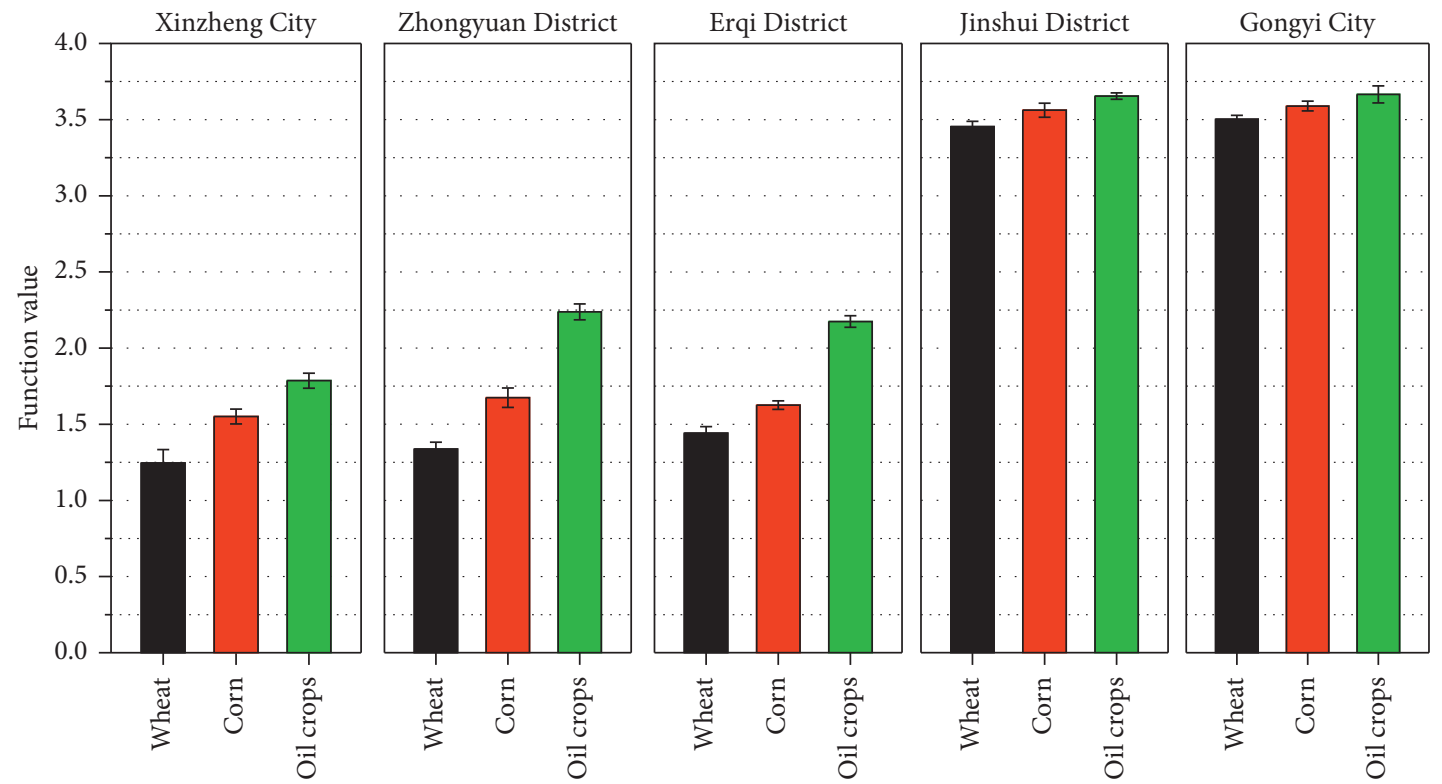

Figure 8: Function values between different crops in different urban areas.

agricultural water resources in the irrigation area. The highefficiency utilization of the irrigated area promotes the agricultural development of the irrigation area and obtains the optimal level of agricultural economic management.

\section{Conclusion}

Guided by a class of multiobjective fuzzy linear programming problems with elastic constraints, this paper discussed the mathematical model of fuzzy linear programming in detail and obtained their optimal solutions by analyzing the data between two different functions. Subsequently, this fuzzy linear programming was applied to agricultural economic management, with five urban areas in Zhengzhou as an entry point to verify the results obtained by fuzzy linearity. And the conclusions are as follows:

(1) On the basis of elastic constraints, the $f\left(t_{i}\right)$ and $g \mathrm{~g}\left(t_{0}\right)$ function expressions and their images were obtained by establishing the degree of expansion and contraction, and then then the optimal inequality solution is obtained by solving and decomposing them by fuzzy linear programming.

(2) Fuzzy linear programming could make more accurate predictions for agricultural economic management. Through the calculation of the $f\left(t_{i}\right)$ and $g \mathrm{~g}\left(t_{0}\right)$ function values of the five districts and cities of Zhengzhou, the pull force affecting agricultural economic management was obtained. The results obtained have consistent changing trends and have a good application prospect. In general, fuzzy linear programming can play a good role in agricultural economic management, which provides theoretical and practical support for the application of fuzzy linear programming.

\section{Data Availability}

The data that support the findings of this study are included within the article.

\section{Conflicts of Interest}

The author declares that there are no conflicts of interest or personal relationships that could have appeared to influence the work reported in this paper.

\section{Acknowledgments}

This work was supported by the Research Project of Henan Federation of Social Sciences: Empirical Study on the Impact of Agricultural Science and Technology Application on Agricultural Modernization, SKL-2021-2474, and Research Project of Xinyang Philosophy and Social Science: Research on the Development Strategy of Circulation Industry Based on Rural Consumption Upgrading, 2021JJ036.

\section{References}

[1] K. D. S. Yu, K. B. Aviso, M. A. B. Promentilla, J. R. Santos, and R. R. Tan, "A weighted fuzzy linear programming model in economic input-output analysis: an application to risk management of energy system disruptions," Environment Systems and Decisions, vol. 36, no. 2, pp. 183-195, 2016.

[2] V. T. Doan, F. Massa, T. Tison, and H. Naceur, "Coupling of homotopy perturbation method and kriging surrogate model for an efficient fuzzy linear buckling analysis: application to additively manufactured lattice structures," Applied Mathematical Modelling, vol. 97, no. 4, pp. 602-618, 2021.

[3] M. Akram, I. Ullah, and M. G. Alharbi, "Methods for solving L $\mathrm{R}$-type pythagorean fuzzy linear programming problems with 
mixed constraints," Mathematical Problems in Engineering, vol. 2021, no. 4, 29 pages, Article ID 4306058, 2021.

[4] N. Wang, M. Reformat, W. Yao, Y. Zhao, and X. Chen, "Fuzzy Linear regression based on approximate Bayesian computation," Applied Soft Computing, vol. 97, Article ID 106763, 2020.

[5] K. Peng and X. Bai, "Welfare effects of rural-urban land conversion on different aged land-lost farmers: exemplified in Wuhan city," Chinese Journal of Population Resources and Environment, vol. 14, no. 1, pp. 45-52, 2021.

[6] X. Zhang, Y. Zhu, and X. Chen, "Fuzzy 2D linear discriminant analysis based on sub-image and random sampling for face recognition," International Journal of Pattern Recognition and Artificial Intelligence, vol. 34, no. 1, pp. 2056001.1-2056001.19, 2020.

[7] T.-Y. Chen, "Pythagorean fuzzy linear programming technique for multidimensional analysis of preference using a squared-distance-based approach for multiple criteria decision analysis," Expert Systems with Applications, vol. 164, no. 5, Article ID 113908, 2021.

[8] Y. Ma, J. Wei, C. Li, C. Liang, and G. Liu, "Fuzzy comprehensive performance evaluation method of rolling linear guide based on improved analytic hierarchy process," Journal of Mechanical Science and Technology, vol. 34, no. 7, pp. 2923-2932, 2020.

[9] J. Li, Y. Sun, L. Gong, N. Chai, and Y. Yin, "Multiattribute fuzzy decision evaluation approach and its application in enterprise competitiveness evaluation," Mathematical Problems in Engineering, vol. 2021, no. 1, 11 pages, Article ID 8867752, 2021.

[10] D.-h. Kim, H. Cho, and H.-c. Cho, "Gastric lesion classification using deep learning based on fast and robust fuzzy $\mathrm{C}$-means and simple linear iterative clustering superpixel algorithms," Journal of Electrical Engineering \& Technology, vol. 14, no. 6, pp. 2549-2556, 2019.

[11] Y. Wen, H. Chang, X. Su, and W. Assawinchaichote, "Eventtriggered fuzzy control of repeated scalar nonlinear systems and its application to Chua's circuit system," IEEE Transactions on Circuits and Systems I: Regular Papers, vol. 67, no. 12, pp. 5347-5357, 2020.

[12] M. Xu, S. Liu, Z. Xu, and W. Zhou, "DEA evaluation method based on interval intuitionistic Bayesian network and its application in enterprise logistics," IEEE Access, vol. 7, pp. 98277-98289, 2019.

[13] C. Rojas, J. R. Rodriguez, S. Kouro, and F. Villarroel, "Multiobjective fuzzy-decision-making predictive torque control for an induction motor drive," IEEE Transactions on Power Electronics, vol. 32, no. 8, pp. 6245-6260, 2016.

[14] M. Alemany, A. Esteso, N. Ortiz, and M. del Pino, "Centralized and distributed optimization models for the multifarmer crop planning problem under uncertainty: application to a fresh tomato Argentinean supply chain case study," Computers \& Industrial Engineering, vol. 153, no. 1, Article ID 107048, 2020.

[15] A. Mansoori, M. Eshaghnezhad, and S. Effati, "Recurrent neural network model: a new strategy to solve fuzzy matrix games," IEEE Transactions on Neural Networks and Learning Systems, vol. 30, no. 8, pp. 2538-2547, 2019.

[16] T. G. Crainic, M. Hewitt, F. Maggioni, and W. Rei, "Partial benders decomposition: general methodology and application to stochastic network design," Transportation Science, vol. 55, no. 2, 2021.
[17] G. Wang and J. Peng, "Fuzzy optimal solution of fuzzy number linear programming problems," International Journal of Fuzzy Systems, vol. 21, no. 3, pp. 865-881, 2019.

[18] A. O. Hamadameen and N. Hassan, "A compromise solution for the fully fuzzy multiobjective linear programming problems," IEEE Access, vol. 6, pp. 43696-43711, 2018.

[19] S. H. Nasseri, A. Ebrahimnejad, and B. Y. Cao, "Linear programming with fuzzy variables," Fuzzy Linear Programming: Solution Techniques and Applications, Springer, Cham, Switzerland, 2019.

[20] J. Jiang and Z. P. Chen, "Stability of multistage stochastic programs with quadratic objective functions," Chinese Journal of Engineering Mathematics, vol. 36, no. 2, pp. 198-218, 2019.

[21] S. Taghikhani, F. Baroughi, and B. Alizadeh, "The mean chance conditional value at risk under interval type-2 intuitionistic fuzzy random environment," Soft Computing, vol. 24, no. 3, pp. 9361-9373, 2020.

[22] S. K. Singh and S. P. Yadav, "Intuitionistic fuzzy multi-objective linear programming problem with various membership functions," Annals of Operations Research, vol. 269, no. 1-2, pp. 693-707, 2018.

[23] Y. Luo and J. J. Zhu, "Resilience Strategy optimization for large aircraft supply chain based on probabilistic language QFD," International Journal of Information Systems and Supply Chain Management, vol. 13, no. 4, pp. 1-24, 2020.

[24] A. Al-Qudaimi, "Ishita approach to construct an intervalvalued triangular fuzzy regression model using a novel leastabsolute based discrepancy," Engineering Applications of Artificial Intelligence, vol. 102, Article ID 104272, 2021.

[25] M. Rahaman, S. P. Mondal, S. Alam, N. A. Khan, and A. Biswas, "Interpretation of exact solution for fuzzy fractional non-homogeneous differential equation under the Riemann-Liouville sense and its application on the inventory management control problem," Granular Computing, vol. 6, pp. 953-976, 2021.

[26] S. K. Das, T. Mandal, and S. A. Edalatpanah, "A mathematical model for solving fully fuzzy linear programming problem with trapezoidal fuzzy numbers," Applied Intelligence, vol. 46, pp. 509-519, 2016.

[27] A. Mahmoudi and M. R. Feylizadeh, "A grey mathematical model for crashing of projects by considering time, cost, quality, risk and law of diminishing returns," Grey Systems: Theory and Application, vol. 8, no. 3, pp. 272-294, 2018.

[28] B. Fuller, X. Meng, and L. Reyzin, "Computational fuzzy extractors," Information and Computation, vol. 275, no. 1, Article ID 104602, 2020.

[29] A. E. Yaakoubi, L. Amhaimar, K. Attari, M. H. Harrak, M. E. Halaoui, and A. Asselman, "Non-linear and intelligent maximum power point tracking strategies for small size wind turbines: performance analysis and comparison," Energy Reports, vol. 5, pp. 545-554, 2019.

[30] T. Ma, B. Wang, Z. Zhang, and B. Ai, “A Takagi-Sugeno fuzzymodel-based finite-time $\mathrm{H}$-infinity control for a hydraulic turbine governing system with time delay," International Journal of Electrical Power \& Energy Systems, vol. 132, no. 9, Article ID 107152, 2021.

[31] W.-L. Mao and G.-Y. Liu, "Development of an adaptive fuzzy sliding mode trajectory control Strategy for two-axis PMSMdriven stage application," International Journal of Fuzzy Systems, vol. 21, no. 3, pp. 793-808, 2019.

[32] Z. Zhong, Y. Zhu, M. V. Basin, and H.-K. Lam, "Event-based multirate control of large-scale distributed nonlinear systems subject to time-driven zero order holds," Nonlinear Analysis: Hybrid Systems, vol. 36, no. 11, Article ID 100864, 2020. 
[33] S. Jain and S. Jain, "Fuzzy generalized weak contraction and its application to Fredholm non-linear integral equation in fuzzy metric space," The Journal of Analysis, vol. 29, no. 5, pp. 619-632, 2020. 